\title{
Erratum: Denoising Phase Unwrapping Algorithm for Precise Phase Shifting Interferometry
}

\author{
[J. Korean Phys. Soc. 71, 82 (2017)] \\ DOI: $10.3938 /$ jkps.71.82
}

\author{
Phan Huy Phuc, Hyug-Gyo RheE* and Young-Sik GHim ${ }^{\dagger}$ \\ Center for Space Optics, Korea Research Institute of Standards and Science, \\ Daejeon 34113, Korea, and Department of Science of Measurement, \\ University of Science and Technology, Daejeon 34113, Korea
}

DOI: $10.3938 /$ jkps. 72.1412

This is a revision of the reference list reported in the original article. In order to clear the contribution of the previous work on the incremental breadth-first search (IBFS) method applied to the PUMA algorithm, we add one more reference to the existing reference list, as in this erratum.

Page 83 : In this paper, we propose an algorithm that modifies the Boykov-Kolmogorov (BK) algorithm using the incremental breadth-first search (IBFS) method [27, 28] to find paths from the source to the sink of a graph.

[28] S. Ali, H. Khan, I. Shaik and F. Ali, Int. J. Eng. and Technol. 7, 254 (2015). 